\title{
A Review of Brodie's Theory and a Reinterpretation of the Muscular Relationship between the Stomatognathic System and Posture
}

\section{Collin DW da Costa* and Timothy DJ da Costa}

Department of Dentistry, Dentist at Studio Dentistico Ass. da Costa-de Clrecq, Italy

*Corresponding Author: Collin DW da Costa, Department of Dentistry, Dentist at Studio Dentistico Ass. da Costa-de Clrecq, Italy.
Received: June 09, 2021

Published: July 09, 2021

(C) All rights are reserved by Collin DW da

Costa and Timothy DJ da Costa.

\begin{abstract}
The muscular-physiology of the stomatognathic-postural system is a studied subject since 1940. Starting from the first considerations on the subject made by Dr A.G. Brodie, the authors of this review wanted to deepen the understanding of the connections and function of this complex system, which is made up of different interconnected systems. This review emphasises the importance of the link between mandibular posture and biomechanics and the biomechanics of the cervical spine through 5 main muscle groups: the hyoid muscles, the mastication muscles, the sub-occipital muscles, the cervical extensors and the flexor muscles of the neck. Therefore, the functional unit of the stomatognathic-postural system is a functional regulating unit, while various different systems (digestive, musculoskeletal, respiratory and psychological) carry out part of their activity through it. By Functional Regulating Unit we mean a system that is able to offset various bodily activities carried out within the same muscular-physiological unit of the organism within functional limits and which, without that system, could lead the human body to have an altered balance due to dysfunctions of one or more of these five muscle groups. The collaboration between the dentist and the therapist should, therefore, be based on the restoration of the correct neuro-muscular physiology of this Unit, where the dentist corrects the occlusal system and the therapist the muscular structures (five muscle groups, TMJ and upper cervical spine) system.
\end{abstract}

Keywords: Review; Posture; Stomatognathic System; Balance; Brodie's Theory; Masticatory System; Collaboration Dentist-Therapist

\section{Introduction}

Brodie's theory and the muscular relationship between the stomatognathic system and posture

The anatomical-functional relationship between the cervical spine, mastication and swallowing is of great importance, both in the field of manual therapy and dentistry. In recent years there has been an ever-growing interest in this subject, but it is mainly studied in the dysfunctional and pathological field. Often are many different muscles involved and this makes it difficult to make a diagnosis and the therapeutic approach is even more complex.
The first researcher to study this relationship was Brodie A.G. who, with his famous Brodie's Theory, represents the beginning of the study of the muscular relationship between the stomatognathic system and posture. According to this rheumatologist, the skeletal components of the head and neck are not balanced. Consequently, the musculature is essential to compensate for the imbalances in mass and weight that arise. This lack of balance is due to the fact that the centre of gravity of the head is located anteriorly to the atlantooccipital joints, which correspond to the head's support points on the cervical spine. Therefore, the head tends to fall forward in the 
upright and resting position because most of the weight of the head is located in front of the fulcrum. Consequently, in order to keep the head in a vertical position, the musculature that connects the back of the skull to the cervical section of the spinal column and the shoulder girdle (cervical extensors) must be tensed.

These muscles are able to raise the individual's angle of vision to the horizon. However, this action is counteracted by the group of antagonists located in the anterior region of the neck and skull. These are the flexor muscles of the cervical spine and the sternocleidomastoid muscles. Between these two muscle groups are the swallowing, mastication and sub-occipital muscles, which act in a synergistic or antagonistic way for the first two groups.

Using the Brodie's Theory, it is possible to understand how the anterior neck muscles can be in contact with the cervical section of the spinal column and with the interposed bone and muscle structures [1].

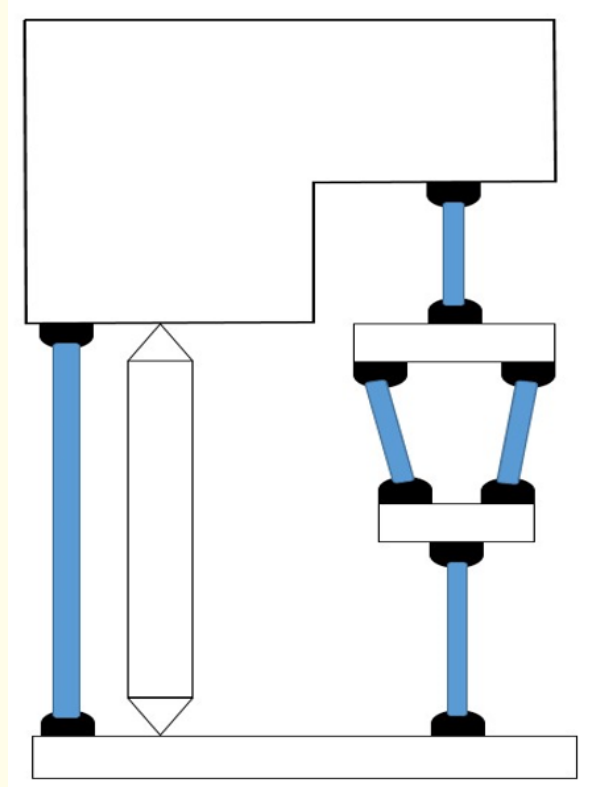

Figure 1: In the image on the side you can see the famous Brodie Theory, which connects the skull posteriorly with the cervical spine and the shoulder girdle and anteriorly with the mandible and the hyoid bone. The muscle groups involved are the cervical extensors, mastication muscles, suprahyoid and infrahyoid muscles.
The authors of this article have decided to use both scientific articles and textbooks that report data provided by scientific arguments. Information published from 1950 up to the recent news published in 2020 is used. There are 37 bibliographic sources and 27 of them are articles published in medical journals. The most consulted scientific journals for this study are the following:

- Journal of Cranio-Mandibular Practice (5 researches);

- Journal of Oral Rehabilitation (4 researches);

For a better understanding of the anatomical-physiological relationship of the stomatognathic-postural system, we have divided this functional unit into 6 subjects:

1. Mastication and swallowing;

2. Mastication and cervical spine muscles;

3. Swallowing-Mastication-Cervical Spine System;

4. Opening and closing the mouth;

5. Swallowing in relation to the cervical spine;

6. Mandibular posture, upper cervical spine and mid-lower cervical spine.

Finally, we tried to integrate the various conclusions in order to be able to reconstruct the various components of the stomatognathic-postural system in a single functional unit so as to understand what they do.

\section{Analysis of the 6 subjects}

The stomatognathic system

If the function of other systems is easy to identify, it is not so easy to understand what the function of the stomatognathic system is. For example, the digestive system's function is to insert food into the body and transform it into energy that can be used by the body itself.

The word "Stomatognathic" is composed of stomato and gnathic. These are two words of Greek origin, where stoma derives from stomato, which means mouth, while gnathic derives from gnatòs, which means mandible [2].

The stomatognathic system is a functional and anatomical unit between the teeth, the maxillary bones, the temporomandibular joints and the mastication muscles [3]. The temporomandibular joints, muscular and ligamentous structures create the direct con- 
nection with the cervical spine, forming the "cranio-cervico-mandibular complex" [4]. The extensive neurological afferents and efferents of the stomatognathic system represented at the level of the large motor and sensory areas of the orofacial area of the cerebral cortex are also part of this complex functional unit [5].

Therefore, by stomatognathic system, we mean that system capable of carrying out all the functions related to the oral cavity and, in particular, the mandible. This includes mastication, swallowing, breathing, speaking, yawning and facial expressions [6].

\section{The relationship between mastication and swallowing}

In 1998 Professor Koole P [7] published an electromyographic study where he analysed the following muscles: temporalis, masseter, medial pterygoid, digastric, mylohyoid and geniohyoid. In this study intramuscular and surface electrodes were applied. The use of intramuscular electrodes allows the interference of other muscles to be removed. However, the biggest problem with this study was the limitation with an intramuscular electrode: rather than all muscle activity being recorded, only part of it was, and that part was unclear. Therefore, during the study, it was necessary to insert a greater number of intramuscular electrodes within the same muscle. Patients had to chew gum first on one side, then on the other side, and finally bilaterally. The test began with the first mastication gum action and finished with the first saliva swallowing action. Registration between one side and the contralateral side was done after 10 minutes to avoid fatigue factors from occurring.

In opening the mouth, the geniohyoid, mylohyoid and digastric muscles, i.e. the swallowing muscles are mainly involved. Meanwhile, in the mouth's closing movements, the temporalis, masseter and medial pterygoid muscles, meaning the masticatory muscles, contract. This demonstrates how these muscles perform an antagonistic effect.

\section{Mastication and cervical spine muscles}

As early as 1970 Funakoshi and Amano [8] published a study where they linked mastication and the cervical spine in mice. They studied the influence of the tonic cervical reflex on the mastication muscles in mice where the labyrinths were destroyed after decerebration. Electrical activity in the mastication muscles increased or decreased in response to rotational or flexion movements of the cervical spine. They concluded that the tonic cervical reflex has an effect on the mastication muscles.
Urbanowicz [9] established that there is a relationship between temporomandibular muscles and joints, on the one hand, and the muscle length and joint position of the occiput and upper cervical spine, on the other. He found that reducing the vertical dimension (Author's Note: followed by a shortening of the mastication muscles) causes a shortening in the suboccipital muscles (reducing the space between the occiput and atlas, therefore extending the occiput on the atlas). This research allows us to understand how hypertonia in the mastication muscles causes hypertonia in the sub-occipital muscles.

\section{The swallowing-mastication-cervical spine system}

In 1983 the physiotherapist Mariano Rocabado [10] wrote in an article: "the position of the hyoid bone is clearly connected to the skull, the mandible and the cervical spine".

In this context, the research published by Valentino B and contributors [11] who was able to demonstrate via an electromyographic examination in 1990, that an alteration of the mandibular posture modifies the function of the cervical, dorsal and lumbar paravertebral muscles, is clearly of interest.

Subsequently, research carried out in 1993 by Esposito and Meersseman [12] made it possible to link the tongue with posture: "an alteration of the position of the tongue, whether resting or in use, not only causes an alteration in the position of the cervical spine and the position of the head, but also on the posture of the scapulohumeral joints. The authors believe that, in this case, the omohyoid muscle is responsible.

The publication of Bracco., et al. [13] in 1998 was also important, as they carried out a study using a stabilometric platform to understand whether changing from centric to myocentric occlusion made positive or negative changes to posture. The conclusion of this publication was that there is a significant improvement in the position of the centre of gravity in the individual analysed.

The research published in 1992 by Huggare J., et al. [14] in this area was crucial, since it was established that there is a relationship between the muscular system of mastication and the muscles that support the head. More specifically, Ohmure H [15] published an article in 2008 where he explained that a forward head posture is associated with retroposition of the mandibular condyle. This conclusion highlights how hypertonia affecting the temporalis 
muscles determines a posterior posture of the mandible, on the one hand, and how, on the other, the anteposition of the head is mediated by an inhibition of the cervical extensors and hypertonia for the sub-occipital muscles, on the other hand, in order to ensure correct vision and thus guarantee balance.

\section{Opening and closing the mouth}

When we try to open our mouths, a small extension at the level of the atlanto-occipital joint is considered physiological. This is also easily imaginable due to the simple fact that the descent of the mandible for opening the mouth leads to a compression of the submandibular tissues, which is not problematic in the initial phase, but becomes complex at the end. For this reason, when opening the mouth, in addition to the mandible descending, an extension of the occiput on the atlas also occurs in order to avoid compressing the submandibular tissues.

This concept is also reiterated by Ranaudo [16] in his text "Riflessioni sulla Lingua" when he states: "both in the extreme opening and in the final part of the oral phase of swallowing, a small extension movement is clinically noted at the level of the upper cervical; that is, of the atlanto-occipital joint. This movement is to be considered physiological".

Research done by Professor Eriksson [17] at the University of Umea in Sweden confirms this assertion. Using a three-dimensional optoelectronic system, he measured the extension movement of the head in the cervical spine during the opening and closing movements of the mouth. The opening of the mouth was always accompanied by an extension of the cervical spine, while its closure was always accompanied by a flexion of the cervical spine. According to this study, the opening movement of the mouth is due to a $50 \%$ extension of the cervical spine, while the remaining $50 \%$ is due to a lowering of the mandible. The results of this research led the author of this study to assume that there is a direct functional connection between the trigeminal nerve and the cervical spine.

To bring the optical plane back to a horizontal position during the closing movement, there is also a slight flexion movement of the cervical spine, which is only the return to the physiological atlanto-occipital position.

It is evident that the sub-occipital muscles play a decisive role in this relationship between the opening and closing movements of the mouth and the flexion-extension of the atlanto-occipital joint.

\section{Swallowing in relation to the cervical spine}

For a correct physiology of the oral phase of swallowing, it is necessary to have not only a good stabilisation of the mandible against the upper maxilla, a good lip seal and a correct posture of the tongue on the palate, but also good stability in the cervical spine and of the head on the neck. The stabilisation of the neck and head by the cervical multifidus muscles and the flexion-extensor muscles of the head and neck is only possible starting from the correct resting posture of the cervical spine and consequent normal values of the atlanto-occipital joint when at rest [18]. The cervical spine reduces the physiological lordosis during the act of swallowing [16] and this occurs through inhibiting the cervical extensors.

Mandibular posture, upper cervical spine and mid-lower cervical spine

The typical conformation of the cervical spine involves its differentiated use:

1. The upper cervical spine (Occiput-C1-C2) These skeletal elements are related to each other by means of a complex articulated joint with three axes and three degrees of freedom;

2. The lower part that goes from the lower plate of the axis to the superior one of the first dorsal vertebra [19].

This consideration also involves a necessary muscular distinction:

1. The sub-occipital muscles that regulate the small adaptive movements in the upper part of the spine and that can determine an extension in the tract between $\mathrm{C} 0-\mathrm{C} 1$ and a rotation of C1 on C2. The superior oblique of the head, the Rectus Minor and Major are used more frequently as postural muscles than as primary movers [20];

2. The cervical extensors, including the superior trapezius, which, if contracted monolaterally, are able to cause a lateral inclination ipsilaterally or, if activated bilaterally, cause an extension of the cervical spine from C3 to C7 [21].

When a person has a deep bite with a consequent mandibular retrusion, one of the main consequences is an inhibition of the extensor muscles of the neck. This is indirectly demonstrated by research conducted by the British Association of Oral and Maxillofacial Surgeons, who noted how the cranio-cervical inclination increased following an anterior correction of the mandible retruded 
in the sagittal plane [22]. The increase in cranio-cervical inclination results in an extension of the occiput over the atlas and requires an increase in the activity of the sub-occipital muscles. A similar result, but in the opposite direction, was also drawn by Moya and Rocabado [23] at the University of Chile in 1994. They used the splint device in 15 subjects and drew two conclusions:

1. A significant reduction in lordosis in the upper part of the cervical spine (the authors speak of C1-C2-C3, but since an extension movement between $\mathrm{C} 1$ and $\mathrm{C} 2$ is not possible, this means that the relationship between the occiput and the atlas is mainly modified and slightly modified even between the second and third cervical vertebrae);

2. An increase in the extension in the rest of the cervical lordosis.

This conclusion involves a fundamental reflection: in relation to the mandibular posture, the sub-occipital and cervical extensors perform an antagonistic function. The mandibular protrusion movement involves a flexion of the occiput on the atlas and an extension from $\mathrm{C} 3$ to $\mathrm{C} 7$, while the mandibular retrusion determines the opposite movement.

\section{Postural-stomatognathic integration}

The preceding work is the basis of the physiology of interaction between the stomatognathic system and the upper part of the cervical spine. Lowering the mandible is mainly mediated by the following muscles: digastric, geniohyoid and the lower belly of the external pterygoid muscle [24]. This implies that the antagonists of these muscles, namely the temporalis, masseter and medial pterygoid, are inhibited and are consequently in a state of hypotonia, acting as antagonist muscles on the former. Similarly, the mastication muscles (except the lower belly of the external pterygoid muscle) can be defined as antagonists of the extensor muscles of the upper part of the cervical spine. Thus, ultimately, the suprahyoid muscles and the cervical extensors can be defined as synergistic, while the masticatory muscles are defined as antagonists [2].

Another group of antagonists at this point is represented by the flexor muscles of the neck, including the sternocleidomastoid. Gadotti I., et al. [25] published an electromyographic study in 2020 where they concluded that a Forward Head Posture, namely, the classic anteposition of the head, determines an increase in activ- ity in the temporalis and masseter muscles. Since this head posture is mainly mediated by hypertonia of the sternocleidomastoid muscles, it can be said that the flexors and mastication muscles are agonists, while the neck flexors and the suprahyoid muscles are antagonists.

Finally, in the mandibular movements, the sub-occipital muscles are antagonists of the cervical extensors. In addition to the research of Lin X [22] and Rocabado [23] mentioned above, this was also demonstrated by researches published by Huggare J [14] and Ohmure H [15].

Having reinterpreted the role of these muscles, which are involved in the stomatognathic system, it is possible to organize them into five groups which, in a coordinated way, cooperate to maintain the individual's balance and other bodily functions, alongside other systems such as vision, vestibular, exteroception and proprioception:

1. Cervical extensor muscles;

2. Masticatory muscles;

3. Suprahyoid muscles;

4. Neck flexor muscles;

5. Sub-occipital muscles.

Therefore, in a condition of ideal neuro-musculo-postural physiology, the activity of only one of these muscle groups simultaneously implies the other groups in this functional unit are cooperating (which can be either through muscle hypertonia or hypotonia). The pivot of the activity of these muscle groups is the temporomandibular joint and the system revolves around the need to keep the gaze horizontal and parallel to the floor, taking into account at the same time the fact that the head naturally tends to fall forward from the greater weight of the head in front of the point of support on the atlas.

On the other hand, the osteo-articular components, which in this system are more susceptible to stresses within this system, are essentially three:

1. Cervical spine and, in particular, the occiput-atlas-axis complex;

2. Intermaxillary relationships: dental occlusion and temporomandibular joints; 
3. Hyoid bone in relation to posture and tongue function.

Any modification of the basic tone of the muscle groups also results in a change in the aforementioned joints.

These considerations allow us to attribute a real adaptive functional modulator role to the stomatognathic system for extra or intra-regional diseases (ascending, descending or mixed factors) and allows us to frame the mechanisms that supervise its regulation (mandibular posture, swallowing, masticatory cycle, occlusion) in the wider field of the laws that regulate postural homeostasis [26].

\section{Stomatognathic system: Balance organ?}

In the study of posture and balance, the stomatognathic system plays a role of fundamental importance. Although the basic principles of posturology are inspired by classical physiology, what changes is the perspective through which they are interpreted. What happens when a body moves through space? And by what means? Is balance only the prerogative of static balance or, in living creatures, must we speak of dynamic balance [27]?

The balance system exerts constant control of the bodily and extracorporeal environment to organise posture: that is, the tonic motor system where voluntary motility occurs [28]. Therefore, it is not easy to completely separate posture and balance and probably not even correct: posture and balance are two interdependent realities, where the first is mainly responsible for statics and the second for dynamics [29].

Here we can better understand the important role of occlusal height in relation to balance. A reduction in the vertical dimension, which is accompanied by a facilitation of the temporalis muscles, leads to an increase in the flexion of the occiput on the atlas, while an increase in the vertical dimension is accompanied by an increase in extension of the occiput on the atlas.

The correlation between balance and the stomatognathic system was highlighted by Esposito and Leisman [30] in 2009 and they indicated that $70 \%$ of the cases examined without problems affecting the cervical spine had repercussions for balance and coordination in opening the mouth to its maximum and closing it tightly.

In 2014, Walczynska-Dragon., et al. [31] conducted a study on the balance and dysfunction of the temporomandibular joint in relation to the mobility of the cervical spine. In this study it was found that the use of the splint device was able to exert a positive effect on the balance of the individual and also on the pain in the cervical spine and on the mobility of this section of the spine.

In this process of opening and closing the mandible, as well as the antigravity response of the masseters as expressed by the study of Miles TS [32], the constant adaptations of the upper cervical spine require reflex responses to maintain the individual's balance.

The publication of Tardieu C., et al. [33] who studied the effect of dental occlusion on posture control in the absence of visual system intervention, is also interesting. Eliminating vision allows all those postural adaptations aimed at always maintaining balance and correct posture to be removed and allows the true effect of dental occlusion and, consequently, the effect of the entire stomatognathic system on the posture of the individual to be truly evaluated. This study allowed several important conclusions to be drawn:

1. Clear effect of dental occlusion on posture;

2. The effect of dental occlusion on posture changes in relation to the static or dynamic condition of the individual;

3. The more the information deriving from proprioception in other systems is reduced as a sensory source to control posture, the greater the effect of dental occlusion on the individual's posture control becomes.

In the context of balance, it is also essential to evaluate the lateral movements of the spine. In 2002, Kibana Y., et al. [34] conducted interesting research at the University of Hokkaido in Japan. Using the electromyograph, a functional difference was found in the right and left sternocleidomastoid muscles in relation to dental occlusion. A unilateral modification causes an imbalance in the physiology of these muscles, consequently causing a lateral inclination of the cervical spine. Meyer P [35] also underlined a correlation between the dental extraction of a molar and the lateral inclination of the average cervical spine, thus hypothesising that a modification of the occlusion can determine an inclination of the cervical spine. This is work that must be further investigated in human physiology and not only in dysfunctionality. However, this publication still allows us to consider the hypothesis that lateral movements of the mandible can in one way or another determine a neuromuscular response to these muscles and therefore play a decisive role in the person's balance. 
Without distinction from the research that has been performed specifically for the lateral inclination movements of the cervical spine in relation to the opening and closing movements of the mandible, it can be hypothesised that a unilateral contraction of the hyoid muscles also causes a contraction of the cervical extensor muscles ipsilaterally. On the basis of what has been asserted (the muscles of mastication and swallowing are antagonists) it can be argued that a contraction of the temporalis muscle determines a lateralisation of the mandible contralaterally [36] and also a lateral inclination of the head towards the opposite side. By uniting the various components of the gnatho-postural system in a single functional unit, the fact remains that a unilateral contraction of the temporalis muscle causes a contralateral mandibular lateralisation with a condylar anteposition on the side of the contracted temporalis muscle through a contraction of the ipsilateral lateral pterygoid muscle [36] and a condylar retroposition on the opposite side mediated by the digastric muscle on that side [37].

This consideration leads to an evaluation of the stomatognathic systems on the three planes and not only on the sagittal or coronal plane, as has been done up to now. The close connection between the various muscle groups involved in the postural stomatognathic system implies an involvement of the vestibular system to maintain the person's balance, based on the mandibular movements first and the cervical spine second. Considering that there are thousands of mandibular movements throughout the day (just think of swallowing, breathing, speaking, eating, etc.), one can easily imagine the close relationship between the stomatognathic system and balance. However, this conclusion highlights that the stomatognathic system is not an organ responsible for balance. Rather, it is a functional regulator which also intervenes in those mechanisms which are involved in the individual's balance.

\section{Conclusion}

A modification to the tone of one of the five muscle groups always leads to an adaptation at the level of the stomatognathic system with a modification to the joint physiology of the temporomandibular joints, occiput-atlas-axis functional unit, mid-inferior cervical spine and the position of the hyoid bone. This relationship always implies an involvement of the organs responsible for balance, and of those involved in the regulation of posture.

However, we can imagine how many times these cooperative mechanisms are called on in the space of a day, where the mouth is also the seat of expression, verbalisation, digestion and breathing". It is here that the authors intend to attribute to the stomatognathic system a real role as a Functional Regulating Unit. The coexistence of numerous fundamental functions for the survival of existence within the same structure implies a capacity for continuous adaptations, without this entailing a renunciation of an essential function such as that of balance. These last considerations are, in the opinion of the authors of this review, very important for those who practice in the field of dentistry. Making changes to the stomatognathic system can have significant repercussions to the person's balance and posture, but also to one or more of the other functions that take place within the stomatognathic system.

For this reason, a complete evaluation of the stomatognathic system in relation to posture must always pass through an evaluation of the mentioned five muscle groups and the osteo-articular components.

\section{Bibliography}

1. Brodie AG. "Anatomy and Physiology of head and neck musculature". American Journal of Orthodontics 36.11 (1950): 831844.

2. Da Costa. "Il Sistema Gnato-Posturale, Maggioli Editore, Santarcangelo di Romagna (RN)” (2020): 118-119.

3. De Leeuw LR and Klasser GD. "Orofacial Pain". Guidelines for Assessment, Diagnosis, and Management, $5^{\text {th }}$ edition. Chicago, IL: Quintessence Publishing Co, Inc (2013).

4. The Academy of Prosthodontics, Glossary of Prosthodontic Terms". Journal of Prosthetic Dentistry 81 (1999): 101.

5. Nakahara H., et al. "Somatosensory-evoked fields for gingiva, lip, and tongue". Journal of Dental Research 83 (2004): 307311.

6. Cuccia A and Caradonna C. "The Relationship Between the Stomatognathic System and Body Posture Clinics 64.1 (2009): 61-66.

7. Koole P. "Masticatory Muscle Function: a Multichannel Electromyographic Investigation, Rijksuniversiteit Groningen (1998). 
8. Funakoshi M and Amano N. "Effects of the Tonic Neck Reflex on the Jaw Muscles of the Rat". Journal of Dental Research 52.4.31 (1973).

9. Urbanowicz M. The Journal of Craniomandibular Practice 9.2 (1991).

10. Rocabado M. "Biomechanical Relationship of the cranial, cervical and hyoid regions". Journal of Craniomandibular Practice 1 (1983): 62-63.

11. Valentino B., et al. "Electromyographic activity of a muscular group in movements specific to boxing". A Journal on Sports Medicine, Sports Traumatology, Exercise Physiology 30.2 (1990): 160-162.

12. Esposito GM and Meersseman JP. "Valutazione della relazione esistente fra l'occlusione e la postura”. Dent Mod 5 (1988): 923-941.

13. Bracco P., et al. "Observations on the correlation between posture and jaw position: a pilot study". Journal of Cranio Mandibular Practice 16.4 (1998): 252-257.

14. Huggare J., et al. "Head Posture and Cervicovertebral and Craniofacial Morphology in Patients with Craniomandibular Dysfunction". Journal of Craniomandibular and Sleep Practice 10.3 (1992): 173-179.

15. Ohmure H., et al. "Influence of forward head posture on condylar position". Journal of Oral Rehabilitation 35.11 (2008): 795-800.

16. Kojiro M., et al. "Cervical Spine motion during swallowing”. European Spine Journal 22.11 (2013): 2558-2563.

17. Erikkson PO and Zafar H. "Concomitant mandibular and headneck movements during jaw opening-closing in man". Journal of Oral Rehabilitation 25.11 (1998): 859-870.

18. Guaglio G. "Bocca e Scoliosi. Nuovi Sviluppi in Ortognatodonzia”. Nike (Orbetello) (2010).

19. Kapandji IA. "Fisiologia Articolare, Tronco e Rachide". Moduzzi Editore (1994): 170.

20. Gray H. "Anatomia del Gray, terza edizione italiana condotta sulla trentasettesima edizione inglese". Zanichelli Editore
(1993): 573.

21. Da Costa T. "Massaggio Posturale, Dall'anatomia alla fisiologia e dalla valutazione posturale ad un innovativo concetto di massaggio". Carta Bianca Editore (2014): 81-82.

22. Lin $X$ and Edwards SP. "Changes in natural head position in response to mandibular advancement". British Journal of Oral and Maxillofacial Surgery 55.5 (2017): 471-475.

23. Moya H., et al. "Influence of Stabilization Occlusal Splint on Craniocervical Relationships. Part I: Cephalometric Analysis". Journal of Craniomandibular Practice 12.1 (1994): 47-51.

24. Grazioli F., et al. "Dall'Otoneurologia alla Posturologia Statica e Dinamica, Valutazione della Sintomatologia Vertiginosa da deficit degli ingressi sensoriali”. Interlinea Editore (2015).

25. Gadotti I., et al. "Electromyography of the masticatory muscles during chewing in different head and neck postures - a pilot study". Journal of Oral Biology and Craniofacial Research 10.2 (2020): 23-27.

26. Ridi R and Saggini R. "Equilibrio Corporeo, Collana di Dinamica Corporea e Benessere". Edizioni Martina 178 (2003): 101.

27. Calasso M. "Analisi Posturale Cranio Cervico Mandibolare: Diagnosi, Terapia e Protocolli Clinici, Corso Teorico-Pratico". Centro Ricerche e Studi Amplifon (2012): 3.

28. Cesarani A. "Disturbi dell'Equilibrio, Medicina del Lavoro e Medicina Sportiva, Springer Verlag Berlin Heidelberg". Arti Grafiche Ricordi (1993): 141.

29. Santandrea G. "Appunti del corso Posturologia Osteopatica, Postural Generation Academy GmbH, Bologna (2017).

30. Esposito V., et al. "Neuromuscolar effects of Temporomandibular joint dysfunction". International Journal of Neuroscience 68.3-4 (1993): 205-207.

31. Walczynska-Dragon Baron S., et al. "Correlation between TMD and Cervical Spine Pain and Mobility: is the whole body balance TMJ related?" BioMed Research International (2014): 582414.

32. Miles TS. "Postural Control of the Human Mandible, University of Adelaide". Archives of Oral Biology 52.4 (2007): 347-352. 
33. Tardieu C., et al. "Dental Occlusion and Postural Control in adults". Neuroscience Letters 450.2 (2009): 221-224.

34. Kibana Y., et al. "Occlusal Support and head posture”. Journal of Oral Rehabilitation 29.1 (2002): 58-63.

35. Meyer PM and Gustowski MS. "Osteopathic Manipulative Treatment to Resolve Head and Neck Pain After Tooth Extraction". Journal of the American Osteopathic Association 112 (2012): 457-460.

36. Fuentes E., et al. "Electromyographic activity of the sphenomandibularis and lateral pterygoid human muscles during mandibular lateral movements". Chirurgia 25 (2012): 97-99.

37. Widmalm SE., et al. "Anatomical and Electromyographic studies of the digastric muscle". Journal of Oral Rehabilitation 15.1

(1988): 3-21.

Volume 5 Issue 8 August 2021

(C) All rights are reserved by Collin DW da Costa and Timothy DJ da Costa. 